\title{
Role of para-cervical block in reducing immediate postoperative pain after total laparoscopic hysterectomy: a prospective randomized placebo-controlled trial
}

\author{
Nilofar Noor, MD, Kallol Kumar Roy, MD, Rinchen Zangmo, MD, Anamika Das, MD, Rakhi Rai, MD, \\ Archana Kumari, MD, Deepali Garg, MD, Sonam Berwa, MBBS, Sushmita Saha, MD, \\ Perumal Vanamail, MSc, PhD \\ Minimally Invasive Unit, Department of Obstetrics and Gynecology, All India Institute of Medical Sciences, New Delhi, India
}

\begin{abstract}
Objective
To study the efficacy and safety of $0.5 \%$ bupivacaine in paracervical block to reduce immediate postoperative pain after total laparoscopic hysterectomy.
\end{abstract}

Methods

A prospective, randomized, double-blind, placebo-controlled study was conducted at a tertiary referral center involving thirty women each in the treatment and placebo groups. Paracervical block with $10 \mathrm{~mL}$ of $0.5 \%$ bupivacaine (treatment group) or $0.9 \%$ saline (placebo group) was administered following general anesthesia and prior to proceeding with total laparoscopic hysterectomy. Visual analogue scale (VAS) scores at 30 and 60 minutes post extubation and mean VAS score (average VAS score at 30 and 60 minutes) were compared. Adequate pain control was defined as mean VAS score $\leq 5$. Additional postoperative opioid requirement, hospital stay, and readmissions were also compared.

Results

Baseline variables such as age, previous history of cesarean section, operating time, and weight of the specimen were comparable in both groups. VAS scores at $30(5.0 \pm 2.8$ vs. $7.0 \pm 1.4)$ and 60 minutes $(5.2 \pm 2.8$ vs. $7.0 \pm 0.8)$ and the mean VAS score $(5.1 \pm 2.7$ vs. $6.8 \pm 0.9)$ were significantly lower in the treatment group. Adequate pain control (mean VAS score $\leq 5)$ was $57 \%$ higher and additional opioid consumption was $47 \%$ lower in the treatment group. No significant difference was found in the duration of hospital stay and readmission rate.

\section{Conclusion}

Paracervical block with bupivacaine was useful in reducing immediate postoperative pain with a $25 \%$ reduction in mean VAS score and a $47 \%$ reduction in opioid consumption in the first hour after total laparoscopic hysterectomy.

Keywords: Bupivacaine; Hysterectomy; Laparoscopy; Nociceptive pain; Pain, postoperative

\section{Introduction}

Laparoscopy has emerged as the preferred mode of surgery for benign gynecological conditions. This minimally invasive approach has many advantages over laparotomy, including early postoperative recovery, early mobilization, and shorter hospital stay [1]. Postoperative pain control is imperative for the realization of all the above benefits of laparoscopy. Inadequate pain relief results in delayed mobilization, which in turn leads to delay in return of bowel function, delayed con-
Received: 2020.09.10. Revised: 2020.10.22. Accepted: 2020.11.19. Corresponding author: Nilofar Noor, MD

Minimally Invasive Unit, Department of Obstetrics and Gynecology, All India Institute of Medical Sciences, Sri Aurobindo Marg, Ansari Nagar, Ansari Nagar East, New Delhi 110029, India E-mail: nilofar.noor@gmail.com

https://orcid.org/0000-0002-8588-7928

Articles published in Obstet Gynecol Sci are open-access, distributed under the terms of the Creative Commons Attribution Non-Commercial License (http://creativecommons. org/licenses/by-nc/3.0/) which permits unrestricted non-commercial use, distribution, and reproduction in any medium, provided the original work is properly cited.

Copyright $\odot 2021$ Korean Society of Obstetrics and Gynecology 


\section{Obstetrics \& Gynecology Science}

Nilofar Noor, et al. Paracervical block in TLH

valescence, and longer hospital stay. In addition, the risk of developing chronic pain may be reduced by early detection and timely treatment of intense acute early postoperative pain, that is, pain within the first 3 days $[2,3]$.

Choi et al. [2] described the characteristics of pain experienced by women undergoing total laparoscopic hysterectomy. They found incisional and visceral pain to be most intense 30 minutes after surgery, gradually decreasing thereafter for the first 72 hours. At all times, visceral pain dominated over incisional pain. Incisional pain had a lower intensity and was described as pricking type, while visceral pain was a dull or heavy inner pain and very intense. Shoulder tip pain was most intense after the first 24 hours.

The pelvic visceral nerve plexus, derived from the hypogastric plexus, is responsible for visceral pain transmission related to the uterus, vagina, bladder, and rectum. Primary innervation of the uterus is caused by the uterosacral plexus, also called the Lee-Frankenhauser plexus. It is located within the uterosacral ligament, lateral to the uterine cervix, and plays a vital role in pain transmission $[4,5]$.

Control of incisional pain and, in particular, visceral pain in the immediate postoperative period is vital for faster convalescence. Multiple strategies have been attempted to reduce postoperative incisional pain after a hysterectomy, such as transverse abdominis plane (TAP) blocks, local anesthetic injected to port sites, and use of a smaller-port caliber [6-9]. Targeted visceral pain control involving the vaginal cuff and pelvic dissection sites are frequently overlooked, even though they contribute significantly to postoperative pain. Systemic analgesics, such as nonsteroidal anti-inflammatory drugs (NSAIDs) and opioids do not directly block visceral pain perception.

Kwack and Kwon [5] suggested that the direct blockade of the Lee-Frankenhauser plexus achieved through the injection of a long-acting analgesic can improve pain relief and enhance recovery. Moreover, by reducing the requirement for systemic analgesics, side effects such as nausea, dizziness, respiratory depression, and opioid addiction may be avoided [5]. Bupivacaine is suitable for targeted block to reduce visceral pain in the immediate postoperative period. Its onset of action is within 15 minutes, and the effect may last for up to 9 hours $[6,10]$. Bupivacaine levels peak in about 30 minutes [11]. Local drug infiltration in the vicinity of the uterosacral nerve plexus has the potential for direct inhibition of neural pain transmission by blocking the pelvic afferent sensory nerve fibers of this plexus [5].

Pain in the immediate postoperative period is acute and most intense and needs to be controlled. Moreover, immediate postoperative pain results in reduced mobilization of respiratory muscles and hypoventilation, causing mucus retention and lung atelectasis, increasing the risk of infection. Pain also results in the release of neurotransmitters and stress hormones that hamper postoperative recovery [5]. Hence, early postoperative pain control is crucial for normal respiration and averting postoperative pulmonary complications [12], as well as for smooth recovery. We aimed to study the efficacy and safety of $0.5 \%$ bupivacaine for paracervical block in the reduction of immediate postoperative pain after total laparoscopic hysterectomy.

\section{Materials and methods}

This was a prospective, randomized, double-blinded, placebo-controlled trial performed in the Minimally Invasive Gynecology Unit at a tertiary referral center with a high surgical case load. Approval from the institute's ethics committee (Ref No. IEC-27 /07.02.2020) was obtained, and the trial was registered under the Clinical Trial Registry-India (CTRI/2020/06/025715). Women in the age group of 25 to 65 years undergoing total laparoscopic hysterectomy for benign gynecological conditions and consenting to participate in the study were included. Concomitant procedures such as salpingo-oophorectomy, endometrioma excision, or ovarian cystectomy were included; however, procedures requiring extensive tissue manipulation and dissection were excluded. Examples of excluded concomitant procedures include pelvic lymph node dissection, sacrocolpopexy, anterior/posterior colporrhaphy, sling surgeries, and deep infiltrating endometriosis requiring extensive dissection and excision. Women with known allergies to amide anesthetics or to whom a paracervical block could not be given due to anatomic abnormalities, such as absent or flushed cervix, were also excluded. Inadvertent intraoperative injury to the gastrointestinal or urologic tract necessitating repair was also excluded as it would prolong the surgery and result in greater postoperative pain.

The calculated sample size was 60. A total of thirty numbers were generated randomly between 1 and 60 using Epi Info version 7.0 software developed by Centers for 


\title{
Obstetrics \& Gynecology Science
}

\author{
Vol. 64, No. 1, 2021
}

Disease Control and Prevention, Atlanta, GA, USA (CDC, Epi Info ${ }^{T M}$ V7, 2011), in such a way that an equal number of patients (30 per group) were allocated to each arm. The random numbers generated were assigned to the treatment group, and the remaining thirty numbers were allocated to the placebo group. The allocation process was maintained by the statistician involved in the project. Upon admission for surgery, appropriate consent was obtained from the eligible patients who were allocated to either a treatment or placebo group in a sequential fashion using a masked list generated prior to commencement of the study via computer-generated randomization. The consenting participants were assigned a serial number sequentially from 1 to 60 along with a unique patient identification number and referred to the statistician who allocated a particular group to the patient based on the random number list. A numerically sequenced, sealed, and opaque envelope containing the assigned group based on the original randomization list as well as the unique patient identification number was kept in the patient's file and handed over to a designated nurse in the theater who did not participate in the surgery or data collection. Based on the group allocation described in the envelope, she prepared a 10-mL syringe labelled "Study" containing either $10 \mathrm{~mL}$ of $0.5 \%$ bupivacaine or $10 \mathrm{~mL}$ of $0.9 \%$ saline and placed it on the operating trolley. The nurse kept the envelope with her and handed it over to the research team at the end of the accrual period. The patient, surgeon, anesthetist, and research team were blinded to the allocation.

The intervention was performed under general anesthesia, prior to starting the surgery, in the lithotomy position. The chief surgeon would inject the contents of the "Study" syringe into the cervical stroma, $5 \mathrm{~mL}$ each at the 3 and 9 o'clock positions, with a depth of $2 \mathrm{~cm}$. A Mangeshikar uterine manipulator was then inserted, and surgery was performed. All hysterectomies were performed by a single chief surgeon with expertise in minimally invasive gynecological surgeries using the same operative technique to minimize bias. Cases were performed using a central $12-\mathrm{mm}$ primary trocar, inserted $2 \mathrm{~cm}$ above the umbilicus in the midline using a direct entry technique, and three accessory 5-mm trocars inserted under direct laparoscopic visualization. Intraabdominal pressure was maintained at $12 \mathrm{mmHg}$. Total laparoscopic hysterectomy was performed in a stepwise manner. Colpotomy was performed using ultrasonic energy Harmonic ACE (Ethicon Endo-Surgery, LLC, Cincinnati, OH, USA) and vault closed horizontally using a barbed suture in a running fashion. A local anesthetic (20 mL of $1 \%$ lignocaine solution) was injected at port sites prior to extubation. Extubation time was noted, and the visual analogue scale (VAS) was used to assess pain at 30 and 60 minutes post-extubation. VAS scores were explained to patients preoperatively, with 0 being no pain at all and 10 being the worst pain ever. The average of VAS scores at 30 and 60 minutes was taken as the mean VAS score. Adequate pain control was said to be achieved when the mean VAS score was $\leq 5$, based on a study by Jensen et al. [13], who considered a 100-mm VAS rating of 0 to $4 \mathrm{~mm}$ as no pain, 5 to $44 \mathrm{~mm}$ as mild, 45 to $74 \mathrm{~mm}$ as moderate, and 75 to $100 \mathrm{~mm}$ as severe pain. All patients received intravenous paracetamol 1 gram $6^{\text {th }}$ hourly for 24 hours for pain relief in the postoperative period, and additional opioid injections (tramadol $50 \mathrm{mg}$ intramuscular) were given for a VAS score greater than 5 at 30 and/ or 60 minutes. Additional opioid requirement in the first 60 minutes after extubation was recorded. Data collected for each patient included age, BMI, number of previous cesarean section (CS), indication for surgery, other procedures performed, total operating time, final pathology, and weight of specimen. At the end of the accrual period, all envelopes containing the patient group and a unique identification number were obtained from the nurse, and group allocation was attached to the data set. Primary outcome measures were VAS score at 30 and 60 minutes and mean VAS score and adequate pain control, whereas secondary outcome measures included additional opioid requirement, nights spent in hospital, and readmissions.

\section{Statistical methods}

An earlier similar study by Radtke et al. [6] showed that the percentage of patients with pain controlled in the placebo arm was $25 \%$ and the study arm was $71 \%$. Expecting similar results, the adequate sample size calculated in each group for $90 \%$ power at a $5 \%$ level of significance was 30 per group. Therefore, 60 patients were recruited for the study. Data analysis was performed using the statistical software STATA version 12.0 (Stata Statistical Software: Release 12; StataCorp LLC, College Station, TX, USA). Descriptive statistics such as mean, standard deviation, and range values were calculated for normally distributed continuous data. Comparison of mean values between the two groups was performed using Student's " $t$ " independent test. Changes in 


\section{Obstetrics \& Gynecology Science}

Nilofar Noor, et al. Paracervical block in TLH

the VAS score at 30 and 60 minutes within the group were compared using the paired t-test. Mean VAS scores at 30 and 60 minutes were compared between the two groups using Student's t-test. Categorical data were expressed as frequency and percentage values. Comparison of frequency data between the categories was tested using Chi square/ Fischer's exact test, as appropriate. For all statistical tests, a two-sided probability of $P$-value $<0.05$ was considered statistically significant.

\section{Results}

Sixty subjects who met the eligibility criteria were prospectively randomized into two groups: thirty each in the treat- ment and placebo groups (Fig. 1). Age and past history of cesarean delivery were comparable in both groups. The mean age was $44.0 \pm 5.9$ years in the treatment group and $44.1 \pm 5.7$ years in the placebo group $(P=0.965)$. Six $(20 \%)$ women in the treatment group and 4 (13.3\%) women in the placebo group had a previous CS $(P=0.731)$. The mean BMI was $26.9 \pm 4.1 \mathrm{~kg} / \mathrm{m}^{2}$ in the treatment group and $25.2 \pm 1.9$ $\mathrm{kg} / \mathrm{m}^{2}$ in the placebo group. No significant difference was found in the operating time and specimen weight between the two groups. The mean operating time was 4,038.0 \pm 961.8 seconds in the treatment group and 3,730.0 4483.6 seconds in the placebo group $(P=0.123)$. The mean weight of the specimen was $260.3 \pm 200.9$ grams in the treatment group and $267.3 \pm 67.8$ grams in the placebo group $(P=0.857)$ (Table 1). Additional procedures were salpingectomy $(n=48)$,

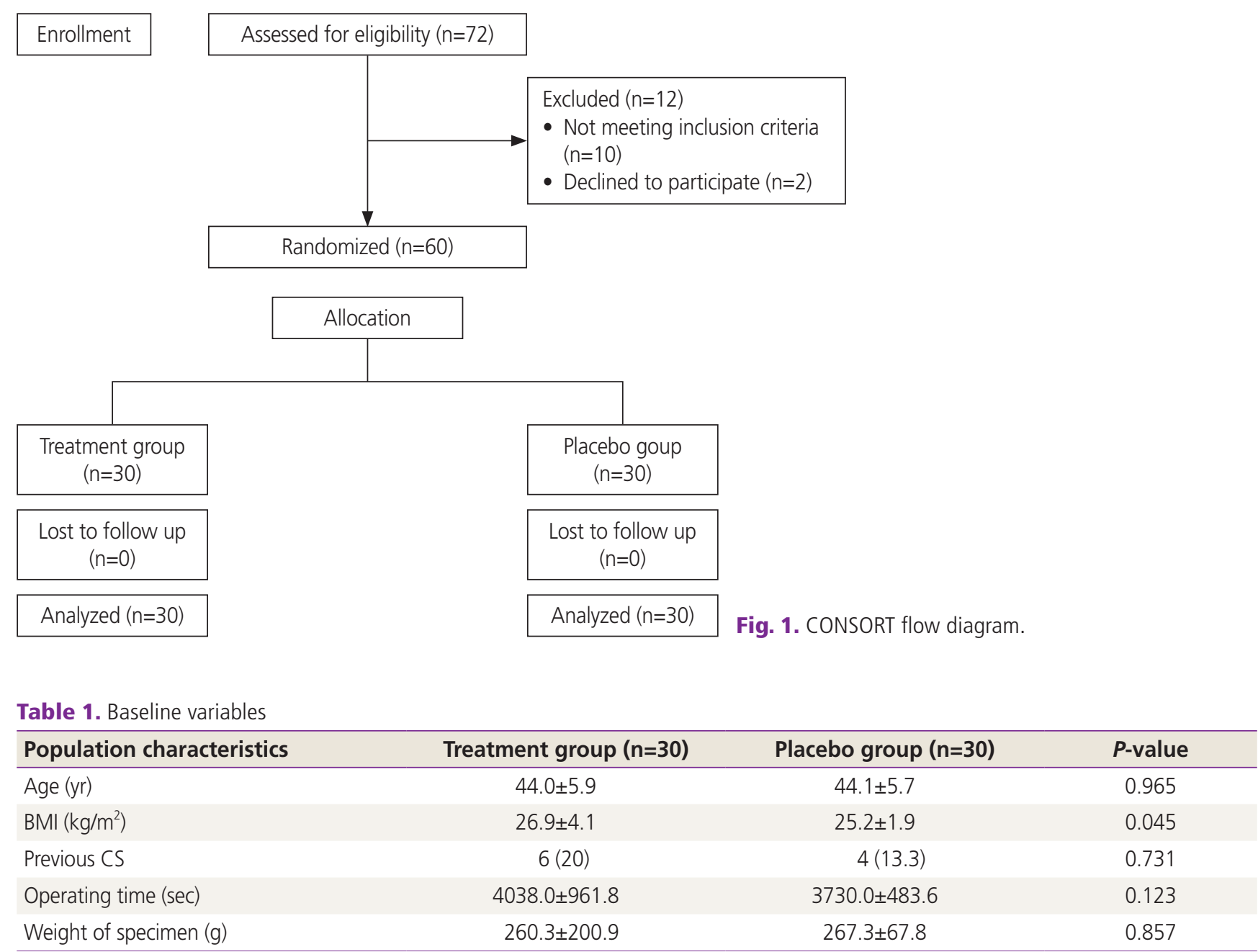

Data were presented as mean \pm standard deviation or number (\%).

BMI, body mass index; CS, cesarean section. 


\title{
Obstetrics \& Gynecology Science
}

\author{
Vol. 64, No. 1, 2021
}

oophorectomy $(n=10)$, and cervical polypectomy $(n=1)$. The most common indication for surgery was heavy menstrual bleeding not responsive to medical management (Table 2), while other indications included persistent postmenopausal bleeding, endometrial hyperplasia with atypia, cervical carcinoma in situ, and high-grade squamous intraepithelial lesion. The most common histopathological diagnosis was leiomyoma ( $n=31,51.7 \%)$, followed by adenomyosis $(n=16$, $26.6 \%)$.

The VAS score during the postoperative period was significantly lower in the treatment group than that in the placebo group, both at 30 minutes and 60 minutes after extubation. The mean VAS score at 30 minutes was $5.0 \pm 2.8$ in the treatment group and $7.0 \pm 1.4$ in the placebo group $(P=0.002)$. The mean VAS score at 60 minutes was $5.2 \pm 2.8$ in the treatment group and $7.0 \pm 0.8$ in the placebo group $(P=0.001)$. Overall, the mean VAS score was $5.1 \pm 2.7$ in the treatment group and $6.8 \pm 0.9$ in the placebo group $(P=0.002)$. The median and interquartile values of the VAS scores at 30 minutes

Table 2. Indications for surgery

\begin{tabular}{lc}
\hline Indication & Values \\
\hline Heavy menstrual bleeding & $50(83.3)$ \\
Postmenopausal bleeding & $1(1.7)$ \\
Endometrial hyperplasia with atypia & $1(1.7)$ \\
Cervical carcinoma in situ & $1(1.7)$ \\
High grade squamous intraepithelial lesion & $7(11.6)$ \\
Total & $60(100)$ \\
\hline
\end{tabular}

Data were presented as number (\%). and 60 minutes and the mean VAS scores between the treatment and placebo groups are depicted in Fig. 2. Hence, a $28.5 \%$ reduction in VAS scores at both 30 and 60 minutes as well as a $25 \%$ reduction in the mean VAS score was achieved in the treatment group compared to placebo. Adequate pain control was achieved in 57\% (17/30) in the treatment group and $0 \%(0 / 30)$ in the placebo group $(P=0.001)$. Additional opioid requirement was significantly greater in the placebo group $(30 / 30,100 \%)$ than that in the treatment group $(16 / 30,53 \%)(P=0.001)$. There was no significant difference in the length of hospital stay, and there were no readmissions in either group. The mean number of nights spent in

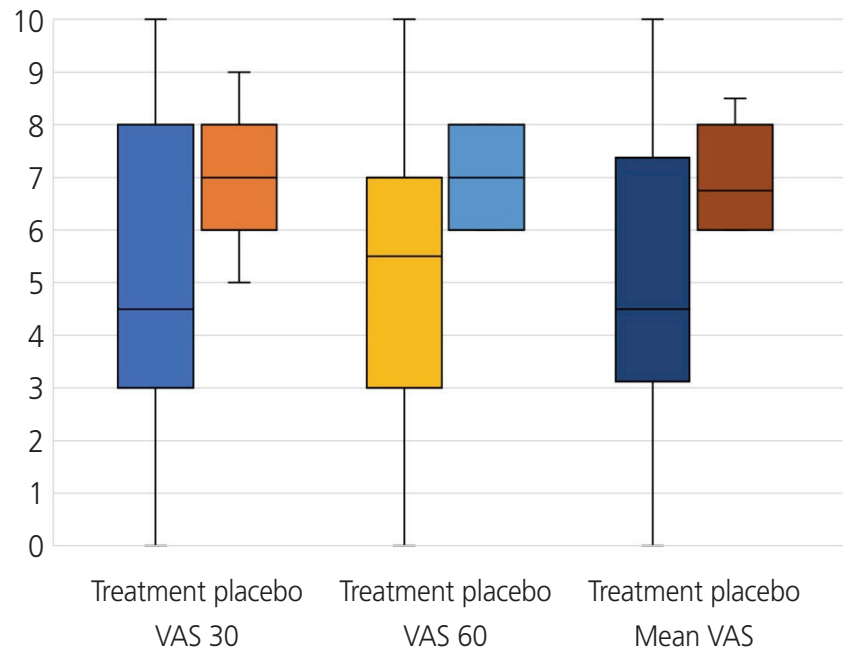

Fig. 2. Boxplot depicting median and interquartile values of visual analogue scale (VAS) scores at 30 minutes and 60 minutes and the mean VAS scores between the treatment and placebo groups.

Table 3. Outcome measures

\begin{tabular}{|c|c|c|c|}
\hline Outcome parameters & Treatment group $(n=30)$ & Placebo group $(n=30)$ & $P$-value \\
\hline \multicolumn{4}{|l|}{ Primary outcomes } \\
\hline VAS score at $30 \mathrm{~min}$ & $5.0 \pm 2.8$ & $7.0 \pm 1.4$ & 0.002 \\
\hline $60 \mathrm{~min}$ & $5.2 \pm 2.8$ & $7.0 \pm 0.8$ & 0.001 \\
\hline Mean VAS score & $5.1 \pm 2.7$ & $6.8 \pm 0.9$ & 0.002 \\
\hline Adequate pain control (mean VAS $\leq 5$ ) & $17(57)$ & $0(0)$ & 0.001 \\
\hline \multicolumn{4}{|l|}{ Secondary outcomes } \\
\hline Additional opioid requirement & $16(53)$ & $30(100)$ & 0.001 \\
\hline Number of nights in hospital & $1.9 \pm 1.3$ & $1.9 \pm 0.8$ & 0.904 \\
\hline Readmission & $0(0)$ & $0(0)$ & - \\
\hline
\end{tabular}

Data were presented as mean \pm standard deviation or number (\%).

VAS, visual analogue scale. 


\section{Obstetrics \& Gynecology Science}

Nilofar Noor, et al. Paracervical block in TLH

the hospital was $1.9 \pm 1.3$ in the treatment group and $1.9 \pm 0.8$ in the placebo group (Table 3).

\section{Discussion}

We found a significant reduction in postoperative pain as well as additional opioid requirement in the immediate postoperative period in patients who received paracervical block with bupivacaine at the initiation of total laparoscopic hysterectomy. Targeted therapy with bupivacaine resulted in a $25 \%$ reduction in the mean VAS score. Adequate pain control in the immediate postoperative period was $57 \%$ greater with bupivacaine, and the need for additional opioid analgesics fell by $47 \%$ in the first 1 hour after surgery. No significant difference was noted in the duration of hospital stay or readmission rate. Hence, paracervical block is an effective method for targeted pain control.

Few studies have attempted to exploit the role of local anesthetic agents in targeted pain therapy for visceral pain in women undergoing hysterectomy. A randomized control study was performed by Radtke et al. [6] in which 21 patients received paracervical block using $0.5 \%$ bupivacaine injection and 20 patients received normal saline as placebo. They demonstrated a significant reduction in the immediate postoperative pain in patients receiving bupivacaine, with $71.4 \%$ of patients achieving successful pain control in the treatment group compared to $25 \%$ in the placebo group. They concluded that the cervix and paracervical tissue could be a promising anatomic location for targeted pain control. Parametrial dissection, creation of the vaginal cuff, and cuff closure contribute significantly to tissue insults during laparoscopic hysterectomy, and access is readily available to these areas by preoperative infiltration of the cervix with local anesthetic [6].

Barr Grzesh et al. [14] performed a randomized, doubleblind, placebo-controlled, parallel-group trial to evaluate the effect of a paracervical block with a combination of bupivacaine and epinephrine before laparoscopic supracervical hysterectomy. They did not find a decrease in the overnight admission rates or postoperative pain, and postoperative opioid use was minimally decreased. However, the authors agree that these results may not be applicable to total hysterectomies.

Blinded, randomized trials by Long et al. [15] and O'Neal et al. [16] also studied the effect of paracervical block with bupivacaine and epinephrine in women undergoing vaginal hysterectomy. Significant reductions in postoperative pain and narcotic use were found in both studies. The mean VAS score at 30 minutes was $43 \%$ lower and at 3 hours was $33 \%$ lower in the bupivacaine group [15].

Hristovska et al. [17] conducted a randomized, placebocontrolled trial on the effect of systemic local infiltration analgesia on postoperative pain in vaginal hysterectomy. Patients received $50 \mathrm{~mL}$ of $0.5 \%$ ropivacaine $(n=20)$ or saline $(n=17)$ infiltration circumferentially around the cervix. They found that systematic infiltration with local ropivacaine in vaginal hysterectomy reduced postoperative pain and opioid intake and facilitated early mobilization and recovery.

In another prospective, double-blind, randomized study, Kwack and Kwon [5] administered $7.5 \%$ ropivacaine or saline into both uterosacral ligaments ( $5 \mathrm{~mL}$ each) transvaginally, prior to closure of the vaginal stump, in women undergoing laparoscopic vaginal hysterectomy. The postoperative pain 2 hours after injection and the analgesic consumption were significantly lower in the ropivacaine group. They also reported that adequate early postoperative pain control minimizes the release of stress hormones and neurotransmitters that interrupt postoperative convalescence.

The strengths of the study include the prospective, randomized, double-blind, placebo-controlled design and an adequate sample size. A single chief surgeon performed all the surgeries, maintaining consistency and reducing bias. However, this also limits the generalizability of the outcome. Past CS may be associated with abdominal adhesions, necessitating greater dissection or increasing the operating time, both of which may result in greater postoperative pain. As both groups were comparable for past CS deliveries, this bias was avoided. Excluding major concomitant pelvic procedures also helped reduce potential confounding factors in pain assessment. The included additional procedures did not require extensive pelvic dissection or significantly prolong the surgery. Hence, they are unlikely to have affected the outcome of the study. Data beyond the first postoperative hour were not collected, which is a limitation of the study. Furthermore, the VAS score is a subjective method of pain assessment that may introduce some bias.

The intensity of visceral pain is maximal in the first few hours after surgery [5], and bupivacaine infiltration is highly effective during the immediate postoperative period and 


\section{Obstetrics \& Gynecology Science}

Vol. 64, No. 1, 2021

targets early pain. Hence, immediate postoperative pain control is of paramount importance for improved quality of postoperative care and recovery. Targeted pain therapy using bupivacaine for paracervical block before total laparoscopic hysterectomy effectively reduces pain and opioid consumption in the immediate postoperative period. Further larger studies are required to reach definitive conclusions.

\section{Conflict of interest}

No potential conflict of interest relevant to this article was reported.

\section{Ethical approval}

Approval from the institute's ethics committee (Ref No. IEC27/07.02.2020) was obtained, and the trial was registered under the Clinical Trial Registry-India (CTRI/2020/06/025715).

\section{Patient consent}

Informed consent was obtained from all study participants.

\section{Funding information}

None.

\section{References}

1. Krishnakumar S, Tambe P. Entry complications in laparoscopic surgery. J Gynecol Endosc Surg 2009;1:4-11.

2. Choi JB, Kang K, Song MK, Seok S, Kim YH, Kim JE. Pain characteristics after total laparoscopic hysterectomy. Int J Med Sci 2016;13:562-8.

3. Bisgaard T, Rosenberg J, Kehlet $\mathrm{H}$. From acute to chronic pain after laparoscopic cholecystectomy: a prospective follow-up analysis. Scand J Gastroenterol 2005;40:135864.

4. Quinn MJ, Kirk N. Differences in uterine innervation at hysterectomy. Am J Obstet Gynecol 2002;187:1515-9.
5. Kwack JY, Kwon YS. Immediate postoperative pain control with ropivacaine following laparoscopic-assisted vaginal hysterectomy: a randomized double-blind pilot study. Taiwan J Obstet Gynecol 2018;57:654-8.

6. Radtke S, Boren T, Depasquale S. Paracervical block as a strategy to reduce postoperative pain after laparoscopic hysterectomy: a randomized controlled trial. J Minim Invasive Gynecol 2019;26:1164-8.

7. Acton JN, Salfinger SG, Tan J, Cohen PA. Outcomes of total laparoscopic hysterectomy using a $5-\mathrm{mm}$ versus 10-mm laparoscope: a randomized control trial. J Minim Invasive Gynecol 2016;23:101-6.

8. Bacal V, Rana U, Mclsaac DI, Chen I. Transversus abdominis plane block for post hysterectomy pain: a systematic review and meta-analysis. J Minim Invasive Gynecol 2019:26:40-52.

9. Charlton S, Cyna AM, Middleton P, Griffiths JD. Perioperative transversus abdominis plane (TAP) blocks for analgesia after abdominal surgery. Cochrane Database Syst Rev 2010:CD007705.

10. Jain S, Nazir N, Singh S, Sharma S. A prospective randomised controlled study for evaluation of high-volume low-concentration intraperitoneal bupivacaine for postlaparoscopic cholecystectomy analgesia. Indian J Anaesth 2018;62:109-14.

11. Hollmén A, Korhonen M, Ojala A. Bupivacaine in paracervical block--plasma levels and changes in maternal and foetal acid-base balance. Br J Anaesth 1969;41:6038.

12. Kelkar KV. Post-operative pulmonary complications after non-cardiothoracic surgery. Indian J Anaesth 2015;59:599-605.

13. Jensen MP, Chen $C$, Brugger AM. Interpretation of visual analog scale ratings and change scores: a reanalysis of two clinical trials of postoperative pain. J Pain 2003:4:407-14.

14. Barr Grzesh RL, Treszezamsky AD, Fenske SS, Rascoff LG, Moshier EL, Ascher-Walsh C. Use of paracervical block before laparoscopic supracervical hysterectomy. JSLS 2018;22:e2018.00023.

15. Long JB, Eiland RJ, Hentz JG, Mergens PA, Magtibay PM, Kho RM, et al. Randomized trial of preemptive local analgesia in vaginal surgery. Int Urogynecol J Pelvic Floor Dysfunct 2009;20:5-10.

16. O'Neal MG, Beste T, Shackelford DP. Utility of preemp- 


\section{Obstetrics \& Gynecology Science}

Nilofar Noor, et al. Paracervical block in TLH

tive local analgesia in vaginal hysterectomy. Am J Obstet Gynecol 2003;189:1539-41.

17. Hristovska AM, Kristensen $B B$, Rasmussen MA, Rasmussen $\mathrm{YH}$, Elving LB, Nielsen CV, et al. Effect of systematic local infiltration analgesia on postoperative pain in vaginal hysterectomy: a randomized, placebo-controlled trial. Acta Obstet Gynecol Scand 2014;93:233-8. 